\title{
Burden of Migraine in Europe Using Self-Reported Digital Diary Data from the Migraine Buddy ${ }^{\complement}$ Application
}

\author{
Pamela Vo · Nicolas Paris · Aikaterini Bilitou • Tomas Valena • \\ Juanzhi Fang • Christel Naujoks • Ann Cameron • Frederic de Reydet de Vulpillieres • \\ Francois Cadiou
}

Received: August 15, 2018 / Published online: October 6, 2018

(C) The Author(s) 2018

\section{ABSTRACT}

Introduction: Migraine is a neurological disease characterized by recurring attacks that can cause severe disabling pain. This study described the burden of migraine as reported by individuals with migraine in the real world using a mobile application.

Methods: A retrospective, cross-sectional analysis was conducted using data captured through the Migraine Buddy@ smartphone application from adult, self-diagnosed individuals with migraine in 17 European countries. Data were analyzed descriptively for the most recent 28-day period reported by users $(n=3900)$

Enhanced digital features To view enhanced digital features for this article go to https://doi.org/10.6084/ m9.figshare.7127204.

P. Vo $(\bowtie)$ · C. Naujoks · F. de Reydet de Vulpillieres Novartis Pharma AG, Basel, Switzerland e-mail: pamela.vo@novartis.com

N. Paris - F. Cadiou

Healint Pte. Ltd, Singapore, Singapore

A. Bilitou

Novartis Global Services Centre, Dublin, Ireland

T. Valena

Novartis s.r.o., Prague, Czech Republic

J. Fang

Novartis Pharma, East Hanover, USA

A. Cameron

Xcenda, Tampa, USA during the study period (June 2015-July 2016) who were randomly selected on the basis of data completeness (completion rates $>70 \%$ ) and stratified by migraine headache days/month: 4-7 episodic migraine (EM; $n=1500), 8-14 \mathrm{EM}$ $(n=1500)$, and chronic migraine $(\geq 15 ; \mathrm{CM}$; $n=900)$.

Results: More than 95\% of users reported that migraine negatively affected their daily activities during at least one migraine attack. Attacks affected $\quad 50.5 \% \quad$ (184.4 days/year), $26.9 \%$ (98 days/year), and $14.5 \%$ (53 days/year) of the year among CM, 8-14 EM, and 4-7 EM groups, respectively. On average, $44.8 \% \mathrm{CM}, 40.9 \%$ 8-14 EM, and $34.7 \%$ of 4-7 EM sufferers, respectively, reported anxiety and/or depression symptoms during migraine attacks. Social or home activities, productivity, and sleep were highly affected, regardless of migraine frequency. Employed respondents $(n=3106)$ reported an average of 2.3 workdays missed per month and that at least one in four migraines led to work absenteeism; these migraines were commonly reported to have at least moderate to severe levels of pain, corresponding to the inability of persons to perform some or even any activities. Triptans (68\%), opioids (46\%), and nonsteroidal anti-inflammatory drugs (45\%) were self-reported as the most common medicines used.

Conclusions: This study, leveraging patient-reported data collected through a mobile application, demonstrates the high burden and 
impact of migraine on health-related quality of life, work productivity, and overall well-being of individuals suffering from migraines.

Funding: Novartis Pharma AG, Switzerland.

Keywords: Burden; Digital application; Europe; Migraine; Migraine Buddy; Real world; Smartphone

\section{INTRODUCTION}

Migraine is a painful, debilitating neurological disease that is often life-long and characterized by recurring attacks. Migraine attacks include headache pain of moderate to severe intensity lasting 4-72 h, often unilateral in pain location, throbbing or pulsating in quality, and worsened by physical activity [1, 2]. Migraine is often associated with symptoms such as nausea, vomiting, phonophobia (sensitivity to sound), and photophobia (sensitivity to light) [1]. The frequency, symptomatology, triggers, and duration of the migraine attacks are also primary considerations used in describing the disease [1]. A single migraine attack typically disrupts the patient's life and can consist of premonitory $(\leq 48 \mathrm{~h})$, aura $(5-60 \mathrm{~min})$, headache $(4-72 \mathrm{~h})$, and resolution/postdrome $(\leq 48 \mathrm{~h})$ phases $[1,3]$. One-third of patients with migraine experience at least 4 headachedays per month, and one-tenth experience at least 15 headache-days per month [4-8].

Migraines are most often treated with acute therapies, which are used to relieve the pain and associated symptoms of a migraine attack at the time it occurs. Prophylactic treatment is meant to reduce the frequency, duration, or severity of migraine attacks. It is recommended for patients in whom acute therapy is either ineffective or contraindicated, in patients with high frequency and severity of migraine, or in patients who suffer significant disability due to aura and other associated symptoms [9, 10]; however, prophylactic treatment is generally underutilized [11, 12].

Globally, migraine affects more than $10 \%$ of the general population [13, 14]. Migraine affects both sexes but is two to three times more prevalent in women than in men $[8,15,16]$.
Migraine prevalence is lower in children/adolescents and those more than 60 years of age [8] and peaks between the ages of 30 and 49 years, during the prime productive years.

Migraine is associated with significant humanistic and economic burden [17-19]. In 2015, migraine was ranked as the seventh leading cause of years lived with disability (YLD) worldwide [18] and in the Global Burden of Disease (GBD) 2016, migraine was the first leading cause of YLD among individuals under 50 years of age $[19,20]$. Migraine was ranked as the fifth leading cause of YLD in Western Europe and the sixth leading cause of YLD in Central and Eastern Europe in the GBD 2015 [21].

Several studies, such as the Eurolight project $[16,20,22]$, the American Migraine Prevalence and Prevention (AMPP) study [23], and the International Burden of Migraine Study (IBMS) [24], have highlighted that migraine has a significant impact on the health-related quality of life (HRQoL) of sufferers and affects their ability to perform home-related, work, and social activities. Migraine has also been found to be associated with depression and anxiety in sufferers [25].

The economic burden of migraine is significant. Different European studies have estimated the cost of migraine at $€ 18$ billion to $€ 27$ billion depending on study setting and design $[17,26,27]$. The majority of all migraine-related costs (77-93\%) are indirect costs attributed primarily to work productivity losses [27, 28].

The objective of the present study was to describe the burden of migraine in terms of impact, symptoms, triggers, and other migraine characteristics as perceived by migraine sufferers in a real-world setting using a self-reported smartphone application (app), Migraine Buddy $\odot$. In today's digital environment, individuals are increasingly using apps to regularly record lifestyle, nutrition, and medical information. Migraine Buddy is the most widely used app by individuals worldwide to record their patterns of migraine, characteristics, and coping mechanisms. The application collects several migraine records across multiple users in several countries; each migraine record corresponds to a migraine attack. 
This study aimed to assess the burden of migraines in terms of affected daily activities, medication usage, and impact of anxiety and depression in patients who suffer from at least four monthly migraine days (MMDs); evaluate the impact of migraine on work productivity; and describe the characteristics of migraine attacks (pain level, duration, symptoms, triggers) experienced by this population of interest.

\section{METHODOLOGY}

\section{Study Design and Data Source}

A retrospective, cross-sectional analysis was conducted using data captured through the smartphone app Migraine Buddy from adults with self-diagnosed migraine in several European countries (UK, France, Spain, Netherlands, Italy, Belgium, Norway, Sweden, Switzerland, Germany, Finland, Poland, Denmark, Portugal, Czech Republic, Hungary, and Slovakia). As noted, Migraine Buddy is a free smartphone app used to collect self-reported migraine data. It is available in four languages (English, French, Japanese, and Spanish). Users are able to regularly log their migraine details into the app, including triggers, symptoms, onset location, migraine frequency and duration, pain intensity and location, use of medication, and impact of migraine on their activities.

\section{Patient Population}

The study used anonymized self-reported data from 3900 randomly selected adult Migraine Buddy users, recorded during the 13-month period from June 1, 2015 through July 3, 2016. The most recent 28-day period reported by each user within the study period was used for analysis. An example of the selection of users and study observation data is presented in Fig. 1.

Data from individuals who were at least 18 years of age, had agreed to participate in the study, and had more than $70 \%$ completeness rates in their migraine records were included in the study sample. Each migraine record describes a single migraine attack. Data from individuals who had used the Migraine Buddy app for less than two consecutive weeks from the time of initial registration, who recorded less than four migraines within a 28-day period, or who skipped the "impact on activity" question for all of the records in scope were excluded. A full list of exclusion criteria and population attrition is presented in Fig. 2, along with the final study sample by country. Users were stratified by migraine frequency as chronic migraine $(\mathrm{CM})$ ( $\geq 15$ headache days/month), episodic migraine (EM) with 4-7 migraine days/month (4-7 EM), and 8-14 migraine days/month (8-14 EM; sometimes referred to as high frequency episodic migraine). Users were randomly selected into the migraine subgroups to fulfill the prespecified convenience sample sizes of 1500 for each of the EM subgroups and 900 for the CM subgroup. Although we present some data on the subgroups, the main focus of this paper is migraine burden among the overall sample of patients who suffer from at least four MMDs. The study was descriptive in nature and thus no formal hypothesis testing was used for sample size calculation. Users were randomly selected from the eligible pool using SciPy, a scientific computing stack for Python.

\section{Study Variables}

The variables examined in the study included the following: demographic characteristics (age, gender); migraine characteristics (triggers, auras, symptoms, pain location and intensity, start time and duration of migraine); migraine burden (location of onset, impact on activities such as social, home, work, medication use, or other relief methods); impact of migraine on work productivity [employment status, workdays missed (absenteeism)]; anxiety and depression; and the number of medications used to treat migraine.

\section{Statistical Analyses}

Demographic variables (age, gender), migraine days per patient, workdays missed, triggers, symptoms, medication usage, and other information were summarized descriptively. As 


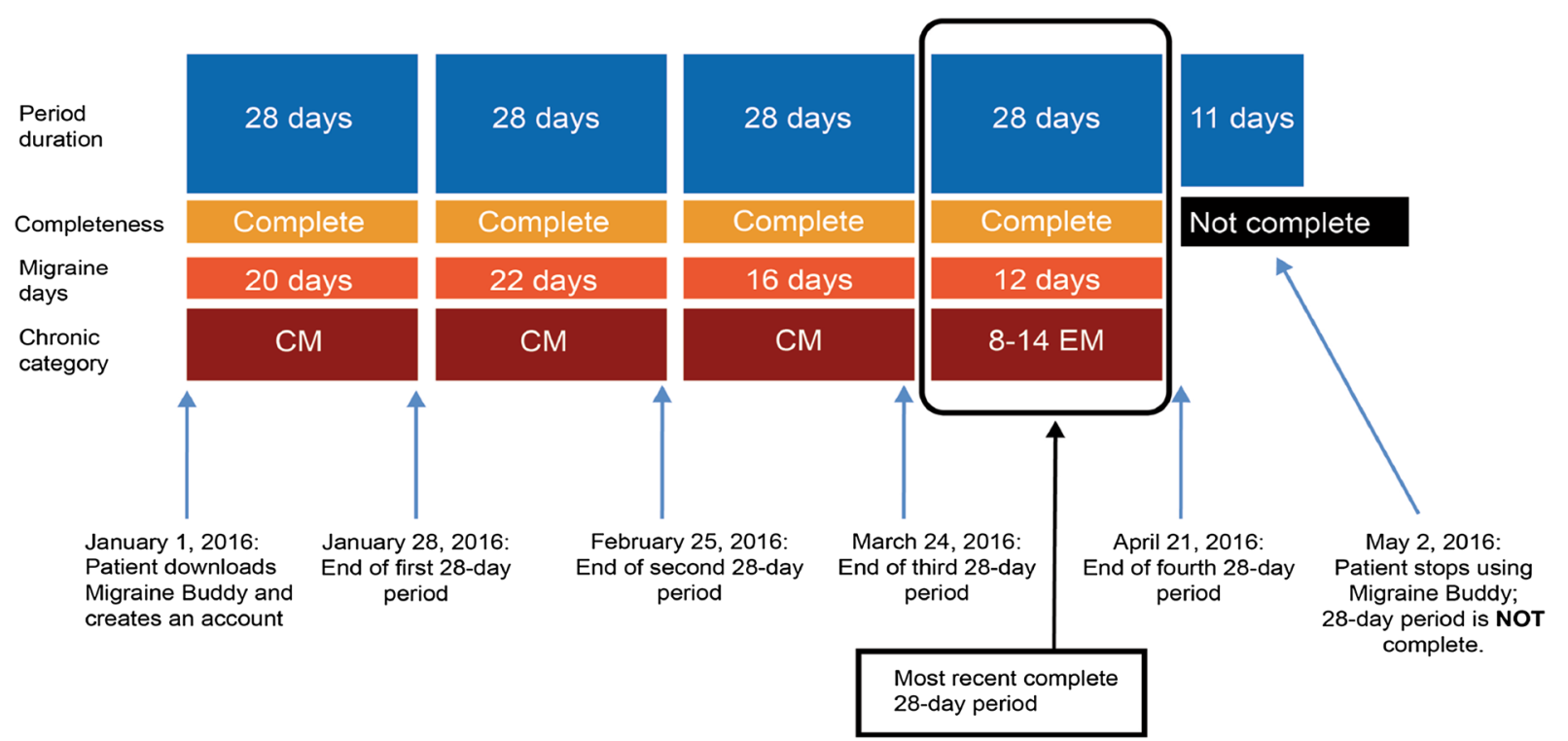

Fig. 1 Example of the selection of eligible Migraine Buddy users and study observation data. $C M$ chronic migraine, $E M$ episodic migraine

noted, some results are presented by migraine frequency subgroup (i.e., 4-7 EM, 8-14 EM, and $\mathrm{CM}$ ) and overall, however, the primary focus of the analyses is the overall sample of patients who suffer from at least four MMDs.

\section{Study Conduct}

The study was conducted in accordance with the Guidelines for Good Pharmacoepidemiology Practices of the International Society for Pharmacoepidemiology [29], the Strengthening the Reporting of Observational Studies in Epidemiology guidelines [30], and with the ethical principles laid out in the Declaration of Helsinki.

This was a non-interventional, retrospective analysis of data collected via individual self-reports through a mobile phone app. Participants provided their authorization for their data to be used for research purposes, and deidentified data were used and analyzed for this study. Patients explicitly confirm that they agree to have their deidentified data used for research purposes; they have the option to refuse and to use the app without any restriction. As such, study subjects were not placed at risk as a result of inclusion in the study sample, and ethics board approval was not warranted.

\section{RESULTS}

\section{Sample Description}

The study sample included 28,152 migraine records (i.e., migraine attacks) from 3900 users who were stratified by migraine frequency into the following subgroups: $4-7 \mathrm{EM}(n=1500)$, 8-14 EM $(n=1500)$, and CM $(n=900)$. Overall, $88 \%$ of users were female, with a reported mean age of 35.4 years (age range 18-74 years with a mean of 10 migraine days per month; a summary of demographics and migraine characteristics of the sample is shown in Table 1). Users were considered to be employed if they reported work as the location of migraine onset or in "affected activities" at least once. In total, 3106 users $(80.0 \%)$ were considered to be employed. Among the overall working population, there was an average of 2.3 workdays missed per month reported during the 28-day observation period, corresponding to $20 \%$ of the overall migraine days experienced. 


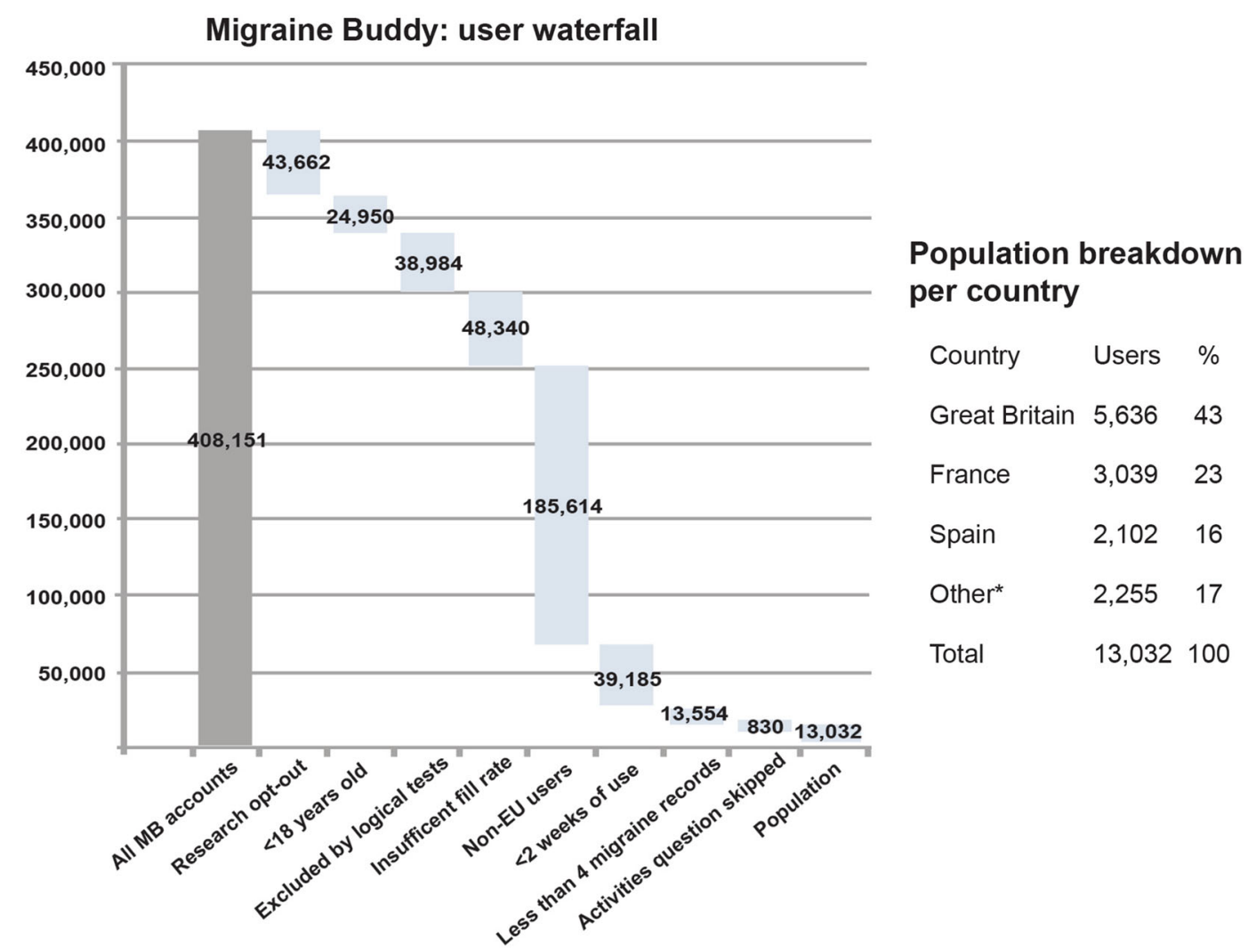

Fig. 2 Waterfall of the Migraine Buddy users by exclusion criteria and study population breakdown by country. $E U$ European Union, $M B$ Migraine Buddy. Others include the following countries: the Netherlands, Italy, Belgium,
Norway, Sweden, Switzerland, Germany, Finland, Poland, Denmark, Portugal, Czech Republic, Hungary, and Slovakia

population reported some type of aura before a migraine attack.

Commonly reported migraine triggers included sleep alterations $(69.5 \%$ of the overall population), psychological factors (65.9\%), nutrition $(55.6 \%)$, menstruation $(52.8 \%)$, and environmental (weather-related) factors $(48.8 \%)$. About $81.0 \%$ of female participants (2059 of 2545 who reported their gender as female) reported menstruation as a trigger of migraine.

The most commonly self-reported migraine symptoms were related to pain/body $(91.7 \%$ of users), mood and cognition (87.3\%, which included nausea, anxiety, confusion, blurred vision, moodiness, or giddiness), and environment [such as ringing in ears (tinnitus), sensitivity to light, noise, or smell; 85.5\%] (each user 
Table 1 Demographics and migraine information of users included in the study

\begin{tabular}{|c|c|c|c|c|}
\hline Characteristics & $\mathrm{CM}(N=900)$ & 8-14 EM $(N=1500)$ & 4-7 EM $(N=1500)$ & Total $(N=3900)$ \\
\hline Gender $(n)^{a}$ & 690 & 1104 & 1087 & 2881 \\
\hline Female $(n, \%)^{\mathrm{a}}$ & $602(87.2)$ & $980(88.8)$ & $963(88.6)$ & $2545(88.3)$ \\
\hline Male $(n, \%)^{\mathrm{a}}$ & $88(12.8)$ & $124(11.2)$ & $124(11.4)$ & $336(11.7)$ \\
\hline Age $(n)^{\mathrm{a}}$ & 478 & 738 & 734 & 1950 \\
\hline Mean $\pm S D^{a}$ & $36.3 \pm 10.4$ & $35.5 \pm 9.9$ & $34.5 \pm 9.4$ & $35.4 \pm 9.9$ \\
\hline Median $^{a}$ & 36.0 & 35.0 & 34.0 & 35.0 \\
\hline Migraine records (total) & 10,347 & 11,301 & 6504 & 28,152 \\
\hline Mean migraine records/patient ${ }^{\mathrm{b}}$ & 11.5 & 7.5 & 4.3 & 7.2 \\
\hline Migraine days (total) & 16,815 & 14,398 & 7693 & 38,906 \\
\hline Mean migraine days/patient ${ }^{\mathrm{c}}$ & 18.7 & 9.6 & 5.1 & 10.0 \\
\hline Working population, $n(\%)^{\mathrm{d}}$ & $730(81)$ & $1237(82)$ & $1139(76)$ & $3106(80)$ \\
\hline Workdays missed (total) & 3216 & 2627 & 1354 & 7197 \\
\hline Mean workdays missed/patient & 4.4 & 2.1 & 1.2 & 2.3 \\
\hline
\end{tabular}

$C M$ chronic migraine, $E M$ episodic migraine, $S D$ standard deviation

${ }^{a}$ Numbers and proportion of respondents who reported gender and/or age in the application during the 28-day study period (June 2015-July 2016) are shown

b Total number of migraine records divided by total number of patients

c Total number of migraine days divided by total number of patients

${ }^{d}$ Working population was considered those who reported work as one of the locations of migraine onset or as an activity affected by migraine

could specify more than one symptom per record and therefore numbers do not add up to $100 \%$ ). Overall, $63.5 \%$ of all migraine records reported a pain intensity greater than or equal to level 5 (on a pain scale of 1-10), corresponding to an inability of persons to perform some or even any activities and indicating a high impact of migraine (Fig. 4). About two out of five individuals with migraine $(39.4 \%$, $n=1537$ ) reported anxiety and/or depression (as a symptom or trigger) during their migraine attack at least once, indicating the high burden of migraine on overall well-being. Anxiety and depression were reported across all migraine frequency subgroups.

\section{Impact of Migraine on Daily Activities}

More than $95 \%$ of users $(n=3732 / 3900$; $1430 / 1500$ users with 4-7 EM, 1447/1500 users with 8-14 EM, and 855/900 users with CM) selfreported that their migraine negatively affected at least one of their daily activities (e.g., home, work or social activities, or sleep) during at least one migraine attack over the observed study period (Table 2).

Migraine can affect all aspects of life for individuals, and results are consistent regardless of whether the user is classified as CM, 4-7 EM, or 8-14 EM. Across migraine records, all aspects of activities were reported to be affected by migraine such as home activities $(32.2 \%$ of records), productivity $(26.7 \%)$, social activities $(18.7 \%)$, and sleep (17.6\%). There was no difference among migraine frequency subgroups in the impact on daily activities and coping mechanisms (such as medication use).

Employed users in the study $(n=3106)$ experienced an average of 10.1 migraine days in the observation period, with about one in four 


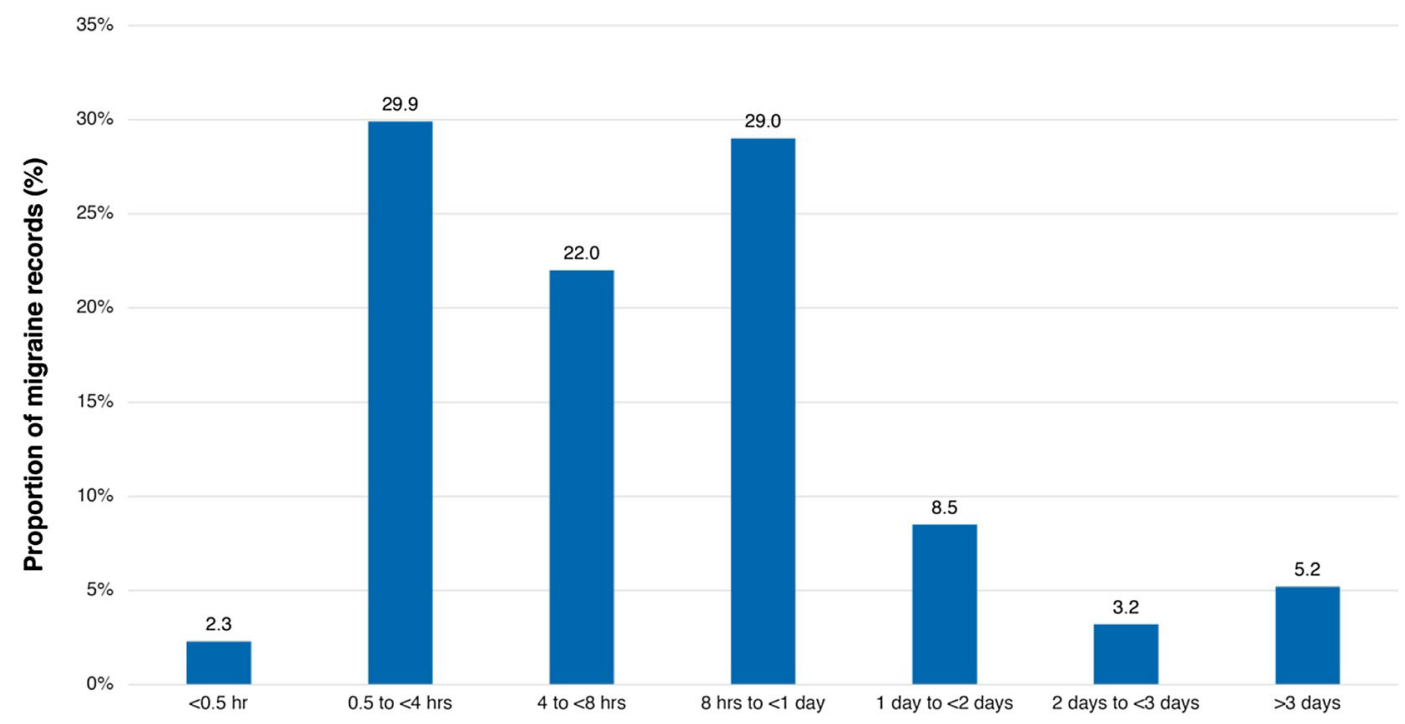

Fig. 3 Duration of migraine attacks reported across migraine records $(n=28,152)$. hr hour

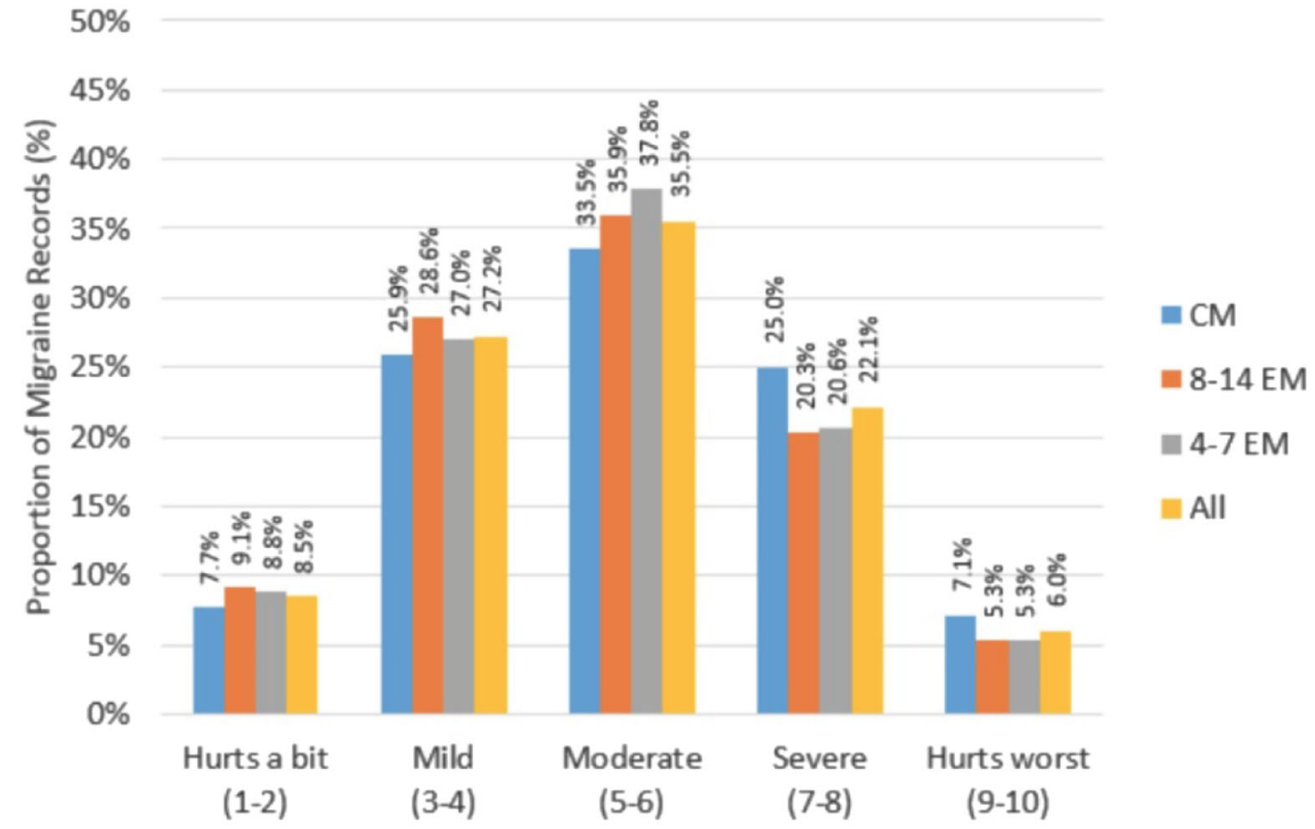

Fig. 4 Pain intensity reported in migraine records $(n=28,152)^{*}$. $C M$ chronic migraine, EM episodic migraine. ${ }^{*}$ Data represents the most recent 28 -day period reported by migraine patients during the study period

migraine days resulting in work absenteeism (average of 2.3 days of missed work). Among employed users, migraines were commonly reported to have at least moderate to severe levels of pain (63\% of migraine records), and
(June 2015-July 2016). Number of migraine records per subgroup: CM, 10,347 records; 8-14 EM, 11,301 records; and 4-7 EM, 6504 records

$83 \%$ of respondents self-reported moderate pain in at least one of their absenteeism-related migraines. The most commonly reported symptoms recorded by migraine sufferers in work absenteeism-related migraines were body 
Table 2 Impact of migraine on users' daily activities as reported in at least one migraine record, by migraine frequency and overall

\begin{tabular}{llccc}
\hline Type of activity affected, $\boldsymbol{n}(\%)$ & $\mathbf{C M}(\boldsymbol{N}=\mathbf{9 0 0})$ & $\mathbf{8 - 1 4}$ EM $(\boldsymbol{N}=\mathbf{1 5 0 0})$ & $\mathbf{4 - 7}$ EM $(\boldsymbol{N}=\mathbf{1 5 0 0})$ & Total $(\boldsymbol{N}=\mathbf{3 9 0 0})$ \\
\hline Home activities & $520(58 \%)$ & $985(66 \%)$ & $933(62 \%)$ & $2438(63 \%)$ \\
Productivity & $590(66 \%)$ & $993(66 \%)$ & $841(56 \%)$ & $2424(62 \%)$ \\
Social activities & $553(61 \%)$ & $882(59 \%)$ & $736(49 \%)$ & $2171(56 \%)$ \\
Sleep & $470(52 \%)$ & $827(55 \%)$ & $676(45 \%)$ & $1973(51 \%)$ \\
Other $^{a}$ & $268(30 \%)$ & $298(20 \%)$ & $204(14 \%)$ & $770(20 \%)$ \\
Any activity & $855(95 \%)$ & $1447(96 \%)$ & $1430(95 \%)$ & $3732(96 \%)$ \\
\hline
\end{tabular}

$C M$ chronic migraine, $E M$ episodic migraine

${ }^{a}$ Other includes affected activities that do not fit in the displayed categories

pain (73\%), mood and cognition (69\%), environmental handicap $(65 \%)$, depression $(24 \%)$, and/or sleep alterations (13\%). There was no difference observed among migraine frequency subgroups on the symptoms recorded by migraine suffers.

\section{Coping with Migraine}

Almost half of all migraine records (47.5\%) reported use of one medication per migraine attack, $28.5 \%$ reported use of two or more medications, and $15.9 \%$ reported no medication use. Overall, $76.1 \%$ of migraine records reported use of at least one drug per migraine record. Triptans $(31.9 \%)$ were the most commonly self-reported acute medication used overall, followed by nonsteroidal anti-inflammatory drugs (NSAIDs) (28.7\%), acetaminophen (18.9\%), and opioids (8.4\%) across migraine records $(n=28,152)$ (Table 3$)$. The medication(s) used during migraine were considered unhelpful in overcoming a migraine attack in $22.0 \%$ of records $(n=7385)$ and helpful in $45.9 \%$ of records $(n=15,410)$. In addition to medication, participants most commonly used rest ( $84.5 \%$ of users, $n=3295$ ) and nutrition (53.0\% of users, $n=2068$ ) as relief methods in at least one migraine attack. Other methods of relief used were ice packs or other non-medication activity that was not captured in the above categories.

\section{DISCUSSION}

Migraine can have a considerable impact on the lives of affected individuals as seen in this sizeable international study, based on self-reported data from individuals suffering from at least four MMDs using a digital phone app. This study highlights the burden associated with migraine attacks on migraine suffers' daily activities, productivity, and well-being. Overall, migraine affected about a third of calendar days $(27.6 \%)$ of the migraine sufferers' lives in this study.

Our study confirms that a migraine attack may last from at least a few hours to days and is often associated with a moderate to severe pain level and other symptoms that affect one or more activities among users, including home responsibilities, social participation, and work. In total, two-thirds of all migraine records reported a pain intensity level of at least 5 (on a scale of 1-10), which can result in being unable to do some activities up to being unable to do any activity because of pain. The burden of migraine is thus substantial and is seen across migraine frequency subgroups.

As a result of the symptom burden, pain level, and duration of migraines, the impact on HRQoL and functioning is considerable [31]. In this study, more than $95 \%$ of the study sample reported that migraine attacks negatively affected their daily activities. Among employed respondents, migraine affected employed respondents' ability to work, with an average of 
Table 3 Types of medications used as recorded across all migraine records

\begin{tabular}{|c|c|c|c|c|}
\hline Type of medication, $n(\%)$ & $\begin{array}{l}\text { CM } \\
(N=10,347)\end{array}$ & $\begin{array}{l}8-14 \mathrm{EM} \\
(N=11,301)\end{array}$ & $\begin{array}{l}4-7 \text { EM } \\
(N=6504)\end{array}$ & $\begin{array}{l}\text { Total } \\
(N=28,152)\end{array}$ \\
\hline NSAIDs (acute treatment) & $2760(26.7)$ & $3259(28.8)$ & $2050(31.5)$ & $8069(28.7)$ \\
\hline Triptans (acute treatment) & $3167(30.6)$ & $4003(35.4)$ & $1823(28.0)$ & $8993(31.9)$ \\
\hline Acetaminophen (acute treatment) & $1825(17.6)$ & $2109(18.7)$ & $1388(21.3)$ & $5322(18.9)$ \\
\hline Opioids (acute treatment) & $1094(10.6)$ & $763(6.8)$ & $514(7.9)$ & $2371(8.4)$ \\
\hline Anti-epileptics (prophylactic treatment) & $703(6.8)$ & $441(3.9)$ & $148(2.3)$ & $1292(4.6)$ \\
\hline $\begin{array}{l}\text { Antihypertension and CCBs (prophylactic } \\
\text { treatment) }\end{array}$ & $455(4.4)$ & $562(5.0)$ & $224(3.4)$ & $1241(4.4)$ \\
\hline Antidepressant (prophylactic treatment) & $280(2.7)$ & $250(2.2)$ & $48(0.7)$ & $578(2.1)$ \\
\hline $\begin{array}{l}\text { Antinausea/antiemetic (symptomatic } \\
\text { treatment) }\end{array}$ & $175(1.7)$ & $160(1.4)$ & $26(0.4)$ & $361(1.3)$ \\
\hline Antihistamine (symptomatic treatment) & $262(2.5)$ & $123(1.1)$ & $53(0.8)$ & $438(1.6)$ \\
\hline Anxiolytics (symptomatic treatment) & $114(1.1)$ & $42(0.4)$ & $14(0.2)$ & $170(0.6)$ \\
\hline Others & $1325(12.8)$ & $1403(12.4)$ & $706(10.9)$ & $3434(12.2)$ \\
\hline No drugs & $1871(18.1)$ & $1597(14.1)$ & $1022(15.7)$ & $4490(15.9)$ \\
\hline
\end{tabular}

"Others" include drugs that do not fit in the displayed categories. "No drugs" means that the patient explicitly said they did not consume any drug. Data represent the most recent 28 -day period reported by migraine patients during the study period (June 2015-July 2015). Users could indicate one or more medications during their migraine and thus numbers may not add up to $100 \%$

$C C B$ calcium channel blocker, $C M$ chronic migraine, $E M$ episodic migraine, NSAID nonsteroidal anti-inflammatory drug

2.3 days of work missed as a result of migraine over the 28-day observation period. This represents an estimated 30.2 workdays missed a year, highlighting the high burden of migraine on work productivity. This is higher than missed workdays reported in the Eurolight project survey, where lost workdays for migraine sufferers averaged about 1 day per month. Differences may be likely due to study samples, as the sample in the Eurolight project included patients with zero to three MMDs [21], whereas our study only included those suffering at least four MMDs. One of the inclusion criteria also required participants to have used the app in at least two consecutive weeks from the time of initial registration before being considered in the study sample, which may have increased the underlying severity of the sample included in our study.
Our findings confirm the substantial burden of migraine found in other global studies such as the Eurolight project and IBMS, despite differences in data collection methodologies and study populations $[16,24,28]$. This is an innovative study utilizing the self-report data from the biggest digital app specific for migraine that allows users to voluntarily record and monitor their migraines as they are occurring and understand their own symptoms, triggers, and migraine characteristics; this has the potential for patients and their caregivers or physicians to better manage their disease but also for the wider scientific community to understand the migraine burden, especially in people of their prime working and reproductive years. The digital app allows for analysis of multiple data from across different countries simultaneously. The data is recorded during the attack or within the hours following the attack, which may 
reduce any memory bias which can occur in studies based on questionnaires.

\section{Limitations}

There are some known limitations inherent to these types of studies that use self-reported data from a digital app. As these data are obtained through a mobile phone app, there is potential bias as the study includes data as reported by users, and no physician diagnosis is used to confirm whether the responder indeed suffers from migraine and the frequency of attacks. We also included data only from users with at least four MMDs, a population that may have higher disease burden and lost productivity (i.e., more missed workdays) than the overall migraine population. Another bias may arise owing to the requirement to have access to a mobile smartphone and use digital apps and, therefore, the population may not be entirely representative of the migraine population in Europe. In addition, a $70 \%$ record completion (fill) rate was used as a data quality check (inclusion criterion) in order to ensure completeness and consistency of data. However, it may be possible that more severe migraines are associated with lower fill rates and thus these users and their migraine records may have been missed from the study sample. The study design, however, allowed the inclusion of a spectrum of migraine frequency and pain levels or durations of migraine attacks.

The design of the study was not focused on gathering feedback on prophylactic medication efficacy and tolerability issues. Since currently available prophylactic medications have been reported to be associated with efficacy and tolerability issues [32-34], this needs to be explored in future research. Effective migraine management may require effective prophylactic treatment for some patients, where physicians and patients consider the scope of migraine-induced disability in addition to migraine symptoms.

Finally, as a result of the short time window of observation period and the lack of historical (longitudinal data) and baseline characteristics (body mass index, comorbidities, social demographics) of application users, there are limitations when interpreting the results and generalizability to other studies of migraine burden and to the general migraine population in Europe.

\section{CONCLUSIONS}

This study provides insights into the real-world burden of migraine on those suffering from at least four MMDs through the collection of patient-recorded, real-time information during and within the hours following the migraine attack. The results highlight the sizeable burden of migraine on the lives of affected individuals in terms of symptoms and impact on daily activities, including work and overall well-being. The large dataset analyzed retrospectively from 3900 users of a popular migraine application has provided results that are comparable and consistent with previous studies published on the burden of migraine in Europe and the rest of the world $[16,29]$.

\section{ACKNOWLEDGEMENTS}

We thank the participants of the study.

Funding. This study was conducted by Healint Pte Ltd and funded by Novartis Pharma AG, Switzerland. The journal processing charges were supported by Novartis Pharma AG, Switzerland. All authors had full access to all of the data in this study and take complete responsibility for the integrity of the data and accuracy of the data analysis.

Medical Writing and Editorial Support. The authors would like to thank Marie-Josee Martel (Xcenda) and Aoife Callan (Novartis) for medical writing support. The authors thank Francois Everhard from Novartis for his assistance in the development of this manuscript.

Authorship. All named authors meet the International Committee of Medical Journal Editors (ICMJE) criteria for authorship for this article, take responsibility for the integrity of the work as a whole, and have given their approval for this version to be published. 
Disclosures. Pamela Vo is a Novartis employee. Tomas Valena is a Novartis employee. Frederic de Reydet de Vulpillieres is a Novartis employee. Juanzhi Fang is a Novartis employee. Christel Naujoks is a Novartis employee. Aikaterini Bilitou was a Novartis employee at the time of the study. Aikaterini Bilitou is an employee of Daiichi Sankyo Europe $\mathrm{GmbH}$. Ann Cameron is an employee of Xcenda. Nicolas Paris is an employee of Healint Pte. Ltd, Singapore. Francois Cadiou is an employee of Healint Pte. Ltd, Singapore.

Compliance with Ethics Guidelines. The study is a retrospective analysis of existing deidentified data from Migraine Buddy users. The study was conducted in accordance with the Guidelines for Good Pharmacoepidemiology Practices of the International Society for Pharmacoepidemiology, the Strengthening the Reporting of Observational Studies in Epidemiology guidelines, and with the ethical principles laid out in the Declaration of Helsinki.

Data Availability. Data sharing is not applicable to this article as the datasets generated and analyzed during the current study are not publicly available for respect of the terms of use of Migraine Buddy, which do not allow the sharing of individual data with a third party.

Open Access. This article is distributed under the terms of the Creative Commons Attribution-NonCommercial 4.0 International License (http://creativecommons.org/licenses/ by-nc/4.0/), which permits any noncommercial use, distribution, and reproduction in any medium, provided you give appropriate credit to the original author(s) and the source, provide a link to the Creative Commons license, and indicate if changes were made.

\section{REFERENCES}

1. International Headache Society. The international classification of headache disorders, 3rd edition (beta version). Cephalalgia. 2013;33(9):629-808.
2. World Health Organization. Headache disorders. Fact sheet $\mathrm{N}^{\circ} 277$. 2016. http://www.who.int/ mediacentre/factsheets/fs277/en/. Accessed $10 \mathrm{Jul}$ 2017.

3. Charles A. The evolution of a migraine attack-a review of recent evidence. Headache. 2013;53:413-9.

4. Blumenfeld AM, Varon SF, Wilcox TK, et al. Disability, HRQoL and resource use among chronic and episodic migraineurs: results from the International Burden of Migraine Study (IBMS). Cephalalgia. 2011;31(3):301-15.

5. Buse DC, Manack A, Serrano D, Turkel C, Lipton RB. Sociodemographic and comorbidity profiles of chronic migraine and episodic migraine sufferers. J Neurol Neurosurg Psychiatry. 2010;81(4):428-32.

6. Buse DC, Manack AN, Fanning KM, et al. Chronic migraine prevalence, disability, and sociodemographic factors: results from the American Migraine Prevalence and Prevention Study. Headache. 2012;52(10):1456-70.

7. Houle TT, Turner DP, Houle TA, et al. Rounding behavior in the reporting of headache frequency complicates headache chronification research. Headache. 2013;53(6):908-19.

8. Lipton RB, Bigal ME, Diamond $\mathrm{M}$, et al. Migraine prevalence, disease burden, and the need for preventive therapy. Neurology. 2007;68(5):343-9.

9. Silberstein S, Holland S, Freitag F, Dodick DW, Argoff C, Ashman E. Evidence-based guideline update: pharmacologic treatment for episodic migraine prevention in adults Report of the Quality Standards Subcommittee of the American Academy of Neurology and the American Headache Society. Neurology. 2012;78:1337-45.

10. Evers S, Afra J, Frese A, et al. EFNS guideline on the drug treatment of migraine-revised report of an EFNS task force. Eur J Neurol. 2009;16(9):968-81.

11. Bloudek LM, Stokes M, Buse DC, et al. Cost of healthcare for patients with migraine in five European countries: results from the International Burden of Migraine Study (IBMS). J Headache Pain. 2012;13:361-78.

12. Sanderson JC, Devine EB, Lipton RB, et al. Headache-related health resource utilisation in chronic and episodic migraine across six countries. J Neurol Neurosurg Psychiatry. 2013;84:1309-17.

13. Stovner LJ, Hagen K, Jensen R, et al. The global burden of headache: a documentation of headache prevalence and disability worldwide. Cephalalgia. 2007;27(3):193-210. 
14. Woldeamanuel YW, Cowan RP. Migraine affects 1 in 10 people worldwide featuring recent rise: a systematic review and meta-analysis of communitybased studies involving 6 million participants. J Neurosci. 2017;372:307-15.

15. Hazard E, Munakata J, Bigal ME, Rupnow MF, Lipton RB. The burden of migraine in the United States: current and emerging perspectives on disease management and economic analysis. Value Health. 2009;12:55-64.

16. Steiner TJ, Stovner LJ, Katsarava Z, et al. The impact of headache in Europe: principal results of the Eurolight project. J Headache Pain. 2014;15(1):31.

17. Stovner LJ, Andree C. Impact of headache in Europe: a review for the Eurolight project. J Headache Pain. 2008;9(3):139-46.

18. Vos T, Allen C, Arora M, et al. Global, regional, and national incidence, prevalence, and years lived with disability for 310 diseases and injuries, 1990-2015: a systematic analysis for the Global Burden of Disease Study 2015. Lancet. 2016;388:1545-602.

19. Steiner TJ, Stovner LJ, Vos T, et al. Migraine is first cause of disability in under 50s: will health politicians now take notice. J Headache Pain. 2018;19:17.

20. Vos T, Abajobir AA, Abbafati C, et al. Global, regional, and national incidence, prevalence, and years lived with disability for 328 diseases and injuries for 195 countries, 1990-2016: a systematic analysis for the Global Burden of Disease Study 2016. Lancet. 2017;390:1211-59.

21. Steiner TJ, Stovner LJ, Vos T. GBD 2015: migraine is the third cause of disability in under 50 s. J Headache Pain. 2016;17(1):104.

22. Andrée C, Stovner LJ, Steiner TJ, et al. The Eurolight project: the impact of primary headache disorders in Europe. Description of methods. J Headache Pain. 2011;12(5):541-9.

23. Munakata J, Hazard E, Serrano D, et al. Economic burden of transformed migraine: results from the American Migraine Prevalence and Prevention (AMPP) Study. Headache. 2009;49:495-508.
24. Payne KA, Varon SF, Kawata AK, et al. The International Burden of Migraine Study (IBMS): study design, methodology, and baseline cohort characteristics. Cephalalgia. 2011;31(10):1116-30.

25. Lampl C, Thomas H, Tassorelli C, et al. Headache, depression and anxiety: associations in the Eurolight project. J Headache Pain. 2016;17:59.

26. Olesen J, Lekander I, Andlin-Sobocki P, Jönsson B. Funding of headache research in Europe. Cephalalgia. 2007;27(9):995-9.

27. Olesen J, Gustavsson A, Svensson M. The economic cost of brain disorders in Europe. Eur J Neurol. 2012;19(1):155-62.

28. Linde M, Gustavsson A, Stovner LJ, et al. The cost of headache disorders in Europe: the Eurolight project. Eur J Neurol. 2012;19(5):703-11.

29. Public Policy Committee. International Society of Pharmacoepidemiology. Guidelines for good pharmacoepidemiology practice (GPP). Pharmacoepidemiol Drug Saf. 2016;25:2-10.

30. Vandenbroucke JP, von Elm E, Altman DG, et al. Strengthening the Reporting of Observational Studies in Epidemiology (STROBE): explanation and elaboration. Epidemiology. 2007;18(6):805-35.

31. Abu Bakar N, Tanprawate S, Lambru G, Torkamani M, Jahanshahi M, Matharu M. Quality of life in primary headache disorders: a review. Cephalalgia. 2016;36(1):67-91.

32. Jackson JL, Cogbill E, Santana-Davila R, et al. A comparative effectiveness meta-analysis of drugs for the prophylaxis of migraine headache. PLoS One. 2015;10:e0130733.

33. Hepp Z, Bloudek LM, Varon SF. Systematic review of migraine prophylaxis adherence and persistence. J Manag Care Pharm. 2014;20(1):22-33.

34. Hepp Z, Rosen NL, Gillard PG, et al. Comparative effectiveness of onabotulinumtoxinA versus oral migraine prophylactic medications on headacherelated resource utilization in the management of chronic migraine: retrospective analysis of a USbased insurance claims database. Cephalalgia. 2016;36:862-74. 